\title{
Structural biology of large molecular complexes - what we learned from the master
}

$\mathrm{Hao} \mathrm{Wu}$

Harvard Medical Sch - Boston, MA, Boston, Massachusetts, United States

As someone who obtained her Ph.D. with the late Prof. Michael Rossmann, I was fortunate to be instilled with the fearlessness towards large molecular complexes and the skills to tackle their structures. In this talk, I will elaborate on our effort in pursing supramolecular complexes in innate immunity, in particular, focusing on inflammasomes which are cytosolic complexes that detect danger signals and activate inflammatory caspases. Active inflammatory caspases in turn process substrates to induce cytokine release and pyroptotic cell death.

One important substrate for inflammatory caspases is a protein known as gasdermin D (GSDMD). This protein has an "inflammatory" N-terminal region (NT) and an inhibitory C-terminal region (CT) with a caspase-cleavable linker in between. Upon proteolytic processing, GSDMD-NT interacts with acidic lipids, oligomerizes and inserts into membranes to form large pores. These GSDMD pores are important for the release, or unconventional protein secretion (UPS), of the IL-1 family cytokines which lack signal sequences, and mediate the inflammatory form of cell death pyroptosis.

In our previously published work $(1,2)$, we found from cryo-EM structure determination that the NT of mouse gasdermin A3 (GSDMA3), a member of the gasdermin family, forms large beta-barrels composed of 104-112 strands from 26-28 subunits. Each NT is composed of a soluble globular region and two membrane-inserted $\beta$-hairpins, and we observed dramatic conformational changes associated with membrane insertion. Further, we identified a positively charged helix that interacts with the acidic lipid.

I will elaborate on our recently determined cryo-EM structure of GSDMD pore composed of 31-35 subunits. Analysis of GSDMD pore and GSDMA3 pore revealed important conformational differences between the two structures, and their modes of lipid interaction. We further show that GSDMD and GSDMA3 both form pre-pores that are oligomerized structures but without the inserted beta strands, and that membrane insertion involves a concerted pre-pore to pore transition. Finally, I will discuss how GSDMD pores may mediate selective release of cytokines.

\section{References}

1 Ruan, J., Xia, S., Liu, X., Lieberman, J. \& Wu, H. Cryo-EM structure of the gasdermin A3 membrane pore. Nature 557, 62-67 (2018).

2 Liu, X. et al. Inflammasome-activated gasdermin D causes pyroptosis by forming membrane pores. Nature 535, 153-158 (2016). 